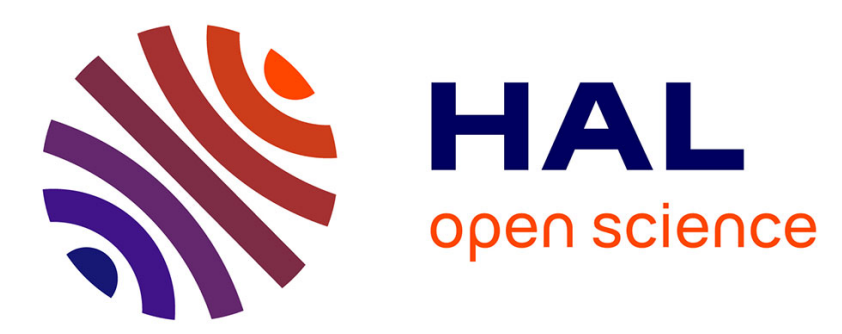

\title{
Robustness of finite-time stability property for sliding modes
}

Emmanuel Bernuau, Andrey Polyakov, Denis Efimov, Wilfrid Perruquetti

\section{To cite this version:}

Emmanuel Bernuau, Andrey Polyakov, Denis Efimov, Wilfrid Perruquetti. Robustness of finite-time stability property for sliding modes. Joint SSSC, TDS, FDA 2013, Feb 2013, Grenoble, France. hal00745673

\section{HAL Id: hal-00745673 \\ https://hal.inria.fr/hal-00745673}

Submitted on 26 Oct 2012

HAL is a multi-disciplinary open access archive for the deposit and dissemination of scientific research documents, whether they are published or not. The documents may come from teaching and research institutions in France or abroad, or from public or private research centers.
L'archive ouverte pluridisciplinaire HAL, est destinée au dépôt et à la diffusion de documents scientifiques de niveau recherche, publiés ou non, émanant des établissements d'enseignement et de recherche français ou étrangers, des laboratoires publics ou privés. 


\title{
Robustness of finite-time stability property for sliding modes
}

\author{
Emmanuel Bernuau* , Andrey Polyakov **, Denis Efimov**, \\ Wilfrid Perruquetti ${ }^{*, * *}$ \\ * LAGIS UMR CNRS 8219, Ecole Centrale de Lille, BP 48, Cité Scientifique, \\ 59651 Villeneuve-d'Ascq, France (e-mail: emmanuel.bernuau@ec-lille.fr). \\ ** Non-A INRIA - LNE, Parc Scientifique de la Haute Borne 40, avenue \\ Halley Bat.A, Park Plaza 59650 Villeneuve d'Ascq, France (e-mail: \\ \{andrey.poliakov, denis.efimov,wilfrid.perruquetti $\} @ i n r i a . f r)$.
}

\begin{abstract}
The paper is devoted to analysis of robustness of the finite-time stability property for discontinuous systems using the homogeneity framework. A short introduction into sliding-mode systems, homogeneity and finite-time stability is given. The main result connects the homogeneity degree of a discontinuous system and its type of robust stability. For robustness analysis the inputto-state stability method is used. The proposed theory is applied to a series of well known sliding-mode control algorithms.
\end{abstract}

Keywords: Sliding-mode Control, Input-to-state stability, Finite-time stability.

\section{INTRODUCTION}

Sliding mode control has a long standing history starting from the early 20th century: the notion of "sliding mode" was introduced in the context of relay control systems. Indeed, the state dependent relay control may switch at high (theoretically infinite) frequency inducing a constraint motion on a manifold (called the sliding mode Utkin [1992]).

Such sliding-mode control exhibits interesting features for engineers: a relative simplicity of design, control equivalent motion (as long as sliding conditions are maintained), invariance to process characteristics and external perturbations Edwards and Spurgeon [1998], Perruquetti and Barbot [2002], Utkin [1992], it still remains the object of many studies (theoretical or related to various applications). Later, the evolution of the sliding mode control theory and its applications required a more detailed specification of the sliding motion, which may have different order of "smothness". The modern control theory calls this extended concept as the high-order sliding-mode (HOSM).

From the beginning, one of the most important properties of the sliding mode was the finite-time convergence of motions toward the sliding manifold. This finite-time convergence property was intensively used within ordinary differential framework Haimo [1986]. Since then, many papers were devoted to the finite-time convergence (Bhat and Bernstein [2005], Moulay and Perruquetti [2003], Orlov [2005]). Until now the main framework, used to obtain FTS (finite-time stability) property, relies upon the notion of homogeneity.

Homogeneity is an intrinsic property of an object, which remains consistent with respect to some scaling: level sets (resp. solutions) are preserved for homogeneous functions (resp. vector fields). The notion of weighted homogeneity was found useful by many authors Hermes [1986], Kawski [1988], Grüne [2000], Nakamura et al. [2009]. Extensions to local homogene-

\footnotetext{
^ This work was partially supported by Non-A INRIA - LNE. ANR Chaslim.
}

ity have been proposed recently Andrieu et al. [2008], Efimov and Perruquetti [2010].

In the context of sliding-mode control and differential inclusions, some few papers were devoted to treatment of FTS by homogeneity Levant [2005], Orlov [2005].

The aim of this paper is to propose a tool to handle the robustness of finite-time stability applying the homogeneity (the advantage of the latter is that it can be checked applying some algebraic operations only). Section 3 introduces the three main ingredients:

- Differential inclusions (DI) within the sliding mode context,

- Finite Time Stability concepts and results for DI,

- Homogeneity concepts and the main results for DI.

Then new results on robustness of FTS for DI with respect to perturbations are given in the context of input-to-state stability Heemels and Weiland [2008], Dashkovskiy et al. [2011] in section 4 , and their consequences for sliding-mode control analysis via the homogeneity are given in section 5 .

\section{NOTATIONS}

Through the paper the following notations will be used:

- $\mathbb{R}_{+}=\{x \in \mathbb{R}: x>0\}, \mathbb{R}_{-}=\{x \in \mathbb{R}: x<0\}$, where $\mathbb{R}$ is the set of real number.

- $\|$. $\|_{i, n}$ denotes the $i$-norm on $\mathbb{R}^{n}$, when $i=2$ (Euclidian norm) it will be simply denoted by $\|$.$\| .$

- For a (Lebesgue) measurable function $d: \mathbb{R}_{+} \rightarrow \mathbb{R}^{p}$ define the norm $\|d\|_{\left[t_{0}, t_{1}\right)}=e s s$. $\sup _{t \in\left[t_{0}, t_{1}\right)}\|d(t)\|$, then $\|d\|_{\infty}=\|d\|_{[0,+\infty)}$ and the set of $d(t)$ with the property $\|d\|_{\infty}<+\infty$ we will further denote as $\mathcal{L}_{\infty}$ (the set of locally essentially bounded measurable functions).

- Let $A, B$ be two subsets of $\mathbb{R}^{n}$, define the Hausdorff distance by 


$$
\|A-B\|_{H}=\max \left\{\sup _{y \in B}\|y\|_{A}, \sup _{x \in A}\|x\|_{B}\right\},
$$

where $\|x\|_{A}=\inf _{y \in A}\|x-y\|$.

- A continuous function $\alpha: \mathbb{R}_{+} \rightarrow \mathbb{R}_{+}$belongs to the class $\mathcal{K}$ if $\alpha(0)=0$ and the function is strictly increasing. The function $\alpha: \mathbb{R}_{+} \rightarrow \mathbb{R}_{+}$belongs to the class $\mathcal{K}_{\infty}$ if $\alpha \in \mathcal{K}$ and it is increasing to infinity. The function $r \in \mathcal{C} \mathcal{L}$ if it is locally Lipschitz continuous in some neighbourhood outside the origin. A continuous function $\beta: \mathbb{R}_{+} \times$ $\mathbb{R}_{+} \rightarrow \mathbb{R}_{+}$belongs to the class $\mathcal{G} \mathcal{K} \mathcal{L}$ if $\beta(s, t) \in \mathcal{K}_{\infty}$ for each fixed $t \in \mathbb{R}_{+}$and $\lim _{t \rightarrow T} \beta(s, t)=0$ for each fixed $s \in \mathbb{R}_{+}$for some $0 \leq T<+\infty$.

- For any $x \in \mathbb{R}^{n}$ the Clarke generalized derivative $D V(x)$ of a locally Lipschitz continuous function $V: \mathbb{R}^{n} \rightarrow \mathbb{R}_{+}$ is defined as follows:

$$
\begin{aligned}
D V(x) & =\left\{\xi: V^{\circ}(x, d) \geq \xi^{\mathrm{T}} d, \forall d \in \mathbb{R}^{n}\right\}, \\
V^{\circ}(x, d) & =\lim _{\inf _{y \rightarrow x, t \rightarrow 0}} \frac{V(y+t d)-V(y)}{t} .
\end{aligned}
$$

The notation $D V(x) f(x)$ stands for directional derivative of a continuously differentiable function $V$ with respect to the vector field $f$ evaluated at point $x$, and for Dini derivative of a Lipschitz continuous function $V$ in the direction of $f$ :

$$
D V(x) f(x)=\lim \inf _{t \rightarrow 0+} \frac{V(x+t f(x))-V(x)}{t} .
$$

- For any real number $\alpha \geq 0$ and for all real $x$ define

$$
\lceil x\rfloor^{\alpha}=\operatorname{sign}(x)|x|^{\alpha} .
$$

\section{PRELIMINARIES}

\subsection{Class of systems under consideration}

In the following we consider ODE of the form

$$
\dot{x}=f(x, d)+g(x) u,
$$

where $x \in \mathbb{R}^{n}$ is the state, $d \in \mathbb{R}^{p}$ is the disturbance, $d(t) \in$ $\mathcal{L}_{\infty}$ and $u$ is the control which belongs to the set of admissible inputs $\mathcal{U} \subset \mathbb{R}^{m}$. Since we are interested in the sliding-mode control analysis, we assume that the control is a discontinuous state feedback $u=u(x)$, which leads to analysis of a closedloop system of the form:

$$
\dot{x}=f_{c l}(x, d),
$$

where $f_{c l}(x, d)=f(x, d)+g(x) u(x)$ is a vector field that is supposed to be at least piecewise continuous. The Filippov regularization procedure allows us to associate a Differential Inclusion (DI) with (2) that captures all behaviours of the system:

$$
\dot{x} \in F_{c l}(x, d),
$$

where $F_{c l}(x, d)$ is a set-valued map. The map $F_{c l}$ satisfies the so-called standard conditions if $F_{c l}$ is compact, convex and upper semi-continuous for all admissible values of its arguments (which is the case when we are considering the Filippov regularization procedure). In this work for an initial condition $x_{0} \in \mathcal{V} \subset \mathbb{R}^{n}$ we denote a corresponding solution defined at least locally in time as $\Phi^{t}\left(x_{0}\right)$ (it is an absolutely continuous function under the standard conditions imposed on $\left.F_{c l}\right)$. The set of solutions $\Phi^{t}\left(x_{0}\right)$ corresponding to the common initial condition $x_{0}$ we will denote as $\mathcal{S}\left(x_{0}\right)$, let $\mathcal{S}=\cup_{x_{0} \in \mathcal{V}} \mathcal{S}\left(x_{0}\right)$ be the set of all possible solutions of the differential inclusion (3) starting in $\mathcal{V}$.

The reader may found additional materials on differential inclusions in Filipov [1988].

\subsection{Finite Time Stability}

The main definitions and properties for FTS are recalled now following the theory emerged in Haimo [1986], Bhat and Bernstein [1998] for the DI (3) with $d=0$.

Definition 1. The system (3) is said to be finite time stable at the origin (on an open neighbourhood $\mathcal{V} \subset \mathbb{R}^{n}$ ) if:

(1) there exists a function $\delta \in \mathcal{K}$ such that for all $x_{0} \in \mathcal{V}$ we have $\left\|\Phi^{t}\left(x_{0}\right)\right\| \leq \delta\left(\left\|x_{0}\right\|\right)$ for all $t \geq 0$ and $\Phi^{t}\left(x_{0}\right) \in$ $\mathcal{S}\left(x_{0}\right)$.

(2) there exists a function $T_{0}: \mathcal{S} \rightarrow \mathbb{R}_{+}$such that for all $x_{0} \in \mathcal{V}$ and $\Phi^{t}\left(x_{0}\right) \in \mathcal{S}\left(x_{0}\right), \Phi^{t}\left(x_{0}\right)=0$ for all $t \geq T_{0}\left[\Phi^{t}\left(x_{0}\right)\right] . T_{0}$ is called the settling-time function of the solution $\Phi^{t}\left(x_{0}\right)$.

If $T(x)=\sup _{\Phi^{t}(x) \in \mathcal{S}(x)} T_{0}\left[\Phi^{t}(x)\right]<+\infty$, then $T$ is called the settling-time function of the system (3).

If $\mathcal{V}=\mathbb{R}^{n}$, then the system is called globally FTS (GFTS).

It is possible to show that if a Lipschitz continuous DI (3) satisfies the standard conditions, then it has the settling-time function $T$ Moulay and Perruquetti [2005].

A sufficient condition of FTS can be formulated for (3) using the Lyapunov theory.

Definition 2. A function $r \in \mathcal{K} \cap \mathcal{C L}$ belongs to class $\mathcal{K} \mathcal{I}$ if there exists $\epsilon>0$ such that:

$$
\int_{0}^{\epsilon} \frac{d z}{r(z)}<+\infty
$$

Theorem 3. Moulay and Perruquetti [2005] Let $0 \in F_{c l}(0)$ in the system (3). If there exists a Lyapunov function $V: \mathbb{R}^{n} \rightarrow$ $\mathbb{R}_{+}$(locally Lipschitz continuous, positive definite and radially unbounded) that verifies the condition

$$
\sup _{\varphi \in F_{c l}(x)} D V(x) \varphi \leq-r[V(x)],
$$

where $r \in \mathcal{K} \mathcal{I}$, then the origin is FTS for (3). Moreover, the settling-time function $T$ of (3) satisfies:

$$
T(x) \leq \int_{0}^{V(x)} \frac{d z}{r(z)} .
$$

A particular possible choice is $r(s)=a s^{\alpha}$, where $a>0$ and $\alpha \in(0,1)$.

\subsection{Homogeneity}

The concept of homogeneity has been introduced in control theory in the 90s Hermes [1986, 1991]. Following ideas of Euler about homogeneous polynomials, Hermes generalized this concept with the weighted homogeneity, applicable to a broader class of systems.

Definition 4. A generalized weight is a vector $\mathbf{r}=\left(r_{1}, \ldots, r_{n}\right)$ with $r_{i}>0$. The dilation associated to the generalized weight $\mathbf{r}$ is the action of the group $\mathbb{R}_{+}^{*}$ on $\mathbb{R}^{n}$ given by:

$$
\begin{aligned}
\Lambda_{\mathrm{r}}: \mathbb{R}_{+}^{*} \times \mathbb{R}^{n} & \longrightarrow \mathbb{R}^{n} \\
(\lambda, x) & \longmapsto \operatorname{diag}\left(\lambda^{r_{i}}\right) x .
\end{aligned}
$$

Many results have been provided for continuous homogeneous systems, usually under the assumption of forward uniqueness of solutions Bhat and Bernstein [2005], Rosier [1992]. Below we present some new results, which have been recently proposed by the authors in the discontinuous setting Bernuau et al. [2013b]. 
Consider the autonomous differential inclusion (3) with $d=0$ defined by the set valued map $F(x)=F_{c l}(x, 0)$ :

$$
\dot{x} \in F(x) \text {. }
$$

Definition 5. A set valued map $F: \mathbb{R}^{n} \rightrightarrows \mathbb{R}^{n}$ is $\mathbf{r}-$ homogeneous with degree $m \in \mathbb{R}$ if for all $x \in \mathbb{R}^{n}$ and for all $\lambda>0$ we have:

$$
\lambda^{-m} \Lambda_{\mathbf{r}}^{-1} F\left(\Lambda_{\mathbf{r}} x\right)=F(x) .
$$

The system (5) is $\mathbf{r}$-homogeneous of degree $m$ if the set valued map $F$ is homogeneous of degree $m$.

Proposition 6. Let $F: \mathbb{R}^{n} \rightrightarrows \mathbb{R}^{n}$ be a set valued $\mathbf{r}$ homogeneous map with degree $m$. Then for all $x_{0} \in \mathbb{R}^{n}$ and all $x($.$) solution of the system (5) with initial condition x_{0}$ and all $\lambda>0$, the absolute continuous curve $t \mapsto \Lambda_{\mathbf{r}}\left(x\left(\lambda^{m} t\right)\right)$ is a solution of the system (5) with initial condition $\Lambda_{\mathbf{r}}\left(x_{0}\right)$.

The homogeneity can be associated with the homogeneous norm (which is a homogeneous function of degree 1)

$$
\|x\|_{r}=\sum_{i=0}^{n}\left|x_{i}\right|^{\rho / r_{i}}, \rho>0 .
$$

Another important object for homogeneous systems is the unit sphere of the homogeneous norm $S_{r}=\left\{x \in \mathbb{R}^{n}:\|x\|_{r}=1\right\}$.

In many situations, the set valued map $F$ comes from the Filippov reguralization process of a discontinuous vector field $f$. Suppose we have a vector field $f$ which is homogeneous in the sense of Definition 5. If we apply the regularization process, is the homogeneity property preserved ? The answer is positive.

Proposition 7. Let $f$ be a vector field and $F$ be the associated set valued map. Suppose $f$ is $\mathbf{r}$-homogeneous of degree $m$. Then $F$ is $\mathbf{r}$-homogeneous of degree $m$.

The following theorem asserts that a strongly globally asymptotically stable system admits a homogeneous Lyapunov function. This result is a generalization of a theorem of Rosier [1992].

Theorem 8. Let $F$ be a $\mathbf{r}$-homogeneous set valued map with degree $m$, satisfying the standard assumptions. Then the two following statements are equivalent:

- The system (5) is strongly globally asymptotically stable.

- For all $k>\max (-m, 0)$, there exist a pair $(V, W)$ of continuous functions, such that:

(1) $V \in C^{\infty}\left(\mathbb{R}^{n}\right), V$ is positive definite and homogeneous with degree $k$;

(2) $W \in C^{\infty}\left(\mathbb{R}^{n} \backslash\{0\}\right), W$ is strictly positive outside of the origin and homogeneous of degree $k+m$;

(3) $\max _{\varphi \in F(x)} D V(x) \varphi \leq-W(x)$ for all $x \neq 0$.

\section{ROBUSTNESS OF FINITE TIME STABILITY PROPERTY}

In this section we will consider the problem of robustness of FTS with respect to external bounded inputs. Mainly the presentation will follow the definitions given in the paper Hong et al. [2010], devoted to an extension of the input-to-state stable (ISS) system theory for the FTS concept. In Hong et al. [2010] all definitions and results are introduced for continuous timevarying nonlinear systems, in this work we will present the extension for DI (3) taking in mind Heemels and Weiland [2008], Xiaowu et al. [2010].

\subsection{Robustness of finite-time stability}

The definition of FTS for (3) with $d=0$ has been given before. In this section we will be interested in two cases for $d \neq 0$ : $d(t)$ is a locally essentially bounded and Lebesgue measurable function of time, i.e. $d \in \mathcal{L}_{\infty}$, and $d(t) \in \mathcal{D}=\left\{d \in \mathcal{L}_{\infty}\right.$ : $\left.\|d\|_{\infty} \leq D\right\}$ for some $0<D<+\infty$. For $d \neq 0$ we will use a notion of extended Filippov's solutions from Heemels and Weiland [2008] and assume that for the chosen class of inputs $\left(\mathcal{D}\right.$ or $\mathcal{L}_{\infty}$ ) the solutions in $\mathcal{S}$ are defined for all $t \geq 0$. Then we have the following list of robust stability properties.

Definition 9. The system (3) is called uniformly FTS at the origin with respect to $d(t) \in \mathcal{D}$ (in an open neighbourhood $\mathcal{V})$ if the following properties hold for all $d(t) \in \mathcal{D}$ :

- uniform stability, i.e. there exists $\delta \in \mathcal{K}$ such that $\left\|\Phi^{t}\left(x_{0}\right)\right\| \leq \delta\left(\left\|x_{0}\right\|\right)$ for all $t \geq 0$, any $x_{0} \in \mathcal{V}$ and all $\Phi^{t}\left(x_{0}\right) \in \mathcal{S}\left(x_{0}\right)$;

- uniform finite-time attractiveness, i.e. there is a settlingtime function $T_{0}: \mathcal{S} \rightarrow \mathbb{R}_{+}$such that $\Phi^{t}\left(x_{0}\right)=0$ for all $t \geq T_{0}\left(\Phi^{t}\left(x_{0}\right)\right)$ for any $x_{0} \in \mathcal{V}$ and all $\Phi^{t}\left(x_{0}\right) \in \mathcal{S}\left(x_{0}\right)$.

If $T(x)=\sup _{\Phi^{t}(x) \in \mathcal{S}(x)} T_{0}\left[\Phi^{t}(x)\right]<+\infty$, then $T$ is called uniform settling-time function of the system (3). The system (3) with $d(t) \in \mathcal{D}$ is called globally uniformly FTS at the origin if $\mathcal{V}=\mathbb{R}^{n}$.

Definition 10. The system (3) is called locally finite-time inputto-state stable (FTISS) if there exist some $\mathcal{D}$ and $\mathcal{V} \subset \mathbb{R}^{n}$, $0 \in \mathcal{V}$ such that for all $x_{0} \in \mathcal{V}$ and $d(t) \in \mathcal{D}$ the estimate

$$
\left\|\Phi^{t}\left(x_{0}\right)\right\| \leq \beta\left(\left\|x_{0}\right\|, t\right)+\gamma\left(\|d\|_{\infty}\right)
$$

is satisfied for all $t \geq 0$ and $\Phi^{t}\left(x_{0}\right) \in \mathcal{S}\left(x_{0}\right)$ for some $\beta \in \mathcal{G K} \mathcal{L}$ and $\gamma \in \mathcal{K}$. The system (3) is called FTISS if $\mathcal{V}=\mathbb{R}^{n}$ and $\mathcal{D}=\mathcal{L}_{\infty}$.

Definition 11. The system (3) is called finite-time integral input-to-state stable (FTiISS) if for all $x_{0} \in \mathbb{R}^{n}$ and $d(t) \in \mathcal{L}_{\infty}$ the estimate

$$
\alpha\left(\left\|\Phi^{t}\left(x_{0}\right)\right\|\right) \leq \beta\left(\left\|x_{0}\right\|, t\right)+\int_{0}^{t} \gamma(\|d(\tau)\|) d \tau
$$

is satisfied for all $t \geq 0$ and $\Phi^{t}\left(x_{0}\right) \in \mathcal{S}\left(x_{0}\right)$ for some $\alpha \in \mathcal{K}_{\infty}$, $\beta \in \mathcal{G K} \mathcal{L}$ and $\gamma \in \overline{\mathcal{K}}$.

The uniform FTS means that the system stability is not influenced by the inputs $d$ from $\mathcal{D}$. The FTISS and FTiISS quantify the deviations of trajectories for bounded and integrally bounded inputs respectively.

The Lyapunov characterization of uniform FTS coincides with one introduced for FTS before, the only additional requirement is that the condition (4) is satisfied for all $d \in \mathcal{D}$. To introduce the Lyapunov functions for FTISS and FTiISS we will need the following property: for two functions $a_{1}, a_{2}: \mathbb{R}^{n} \rightarrow \mathbb{R}_{+}$the relation $a_{1}(x) \sim a_{2}(x)$ means that there exists $\epsilon>0$ such that $a_{1}(x) \geq a_{2}(x)$ for all $\|x\| \leq \epsilon$.

Definition 12. A locally Lipschitz continuous function $V$ : $\mathbb{R}^{n} \rightarrow \mathbb{R}_{+}$is called FTISS Lyapunov function for the system (3) if there are $\alpha_{1}, \alpha_{2}, \alpha_{3} \in \mathcal{K}_{\infty}$ and $\sigma \in \mathcal{K}$ such that for all $x \in \mathbb{R}^{n}$ and $d \in \mathbb{R}^{p}$

$$
\begin{gathered}
\alpha_{1}(\|x\|) \leq V(x) \leq \alpha_{2}(\|x\|), \\
\sup _{\varphi \in F_{c l}(x, d)} D V(x) \varphi \leq-\alpha_{3}(\|x\|)+\sigma(\|d\|)
\end{gathered}
$$

with $\alpha_{3}(\|x\|) \sim a V^{\alpha}(x)$ for some $a>0$ and $0<\alpha<1$. 
As in Heemels and Weiland [2008], Xiaowu et al. [2010] an equivalent FTISS Lyapunov function definition can be used instead of (6):

$$
\|x\| \geq \chi(\|d\|) \rightarrow \sup _{\varphi \in F_{c l}(x, d)} D V(x) \varphi \leq-\alpha_{3}(\|x\|)
$$

for all $x \in \mathbb{R}^{n}$ and $d \in \mathbb{R}^{p}$ with some $\alpha_{3} \in \mathcal{K}_{\infty}, \chi \in \mathcal{K}$.

Definition 13. A locally Lipschitz continuous function $V$ : $\mathbb{R}^{n} \rightarrow \mathbb{R}_{+}$is called FTiISS Lyapunov function for the system (3) if there are $\alpha_{1}, \alpha_{2} \in \mathcal{K}_{\infty}, \sigma \in \mathcal{K}$ and a positive definite continuous function $\alpha_{3}: \mathbb{R}_{+} \rightarrow \mathbb{R}_{+}$such that for all $x \in \mathbb{R}^{n}$ and $d \in \mathbb{R}^{p}$

$$
\begin{gathered}
\alpha_{1}(\|x\|) \leq V(x) \leq \alpha_{2}(\|x\|), \\
\sup _{\varphi \in F_{c l}(x, d)} D V(x) \varphi \leq-\alpha_{3}(\|x\|)+\sigma(\|d\|)
\end{gathered}
$$

with $\alpha_{3}(\|x\|) \sim a V^{\alpha}(x)$ for some $a>0$ and $0<\alpha<1$.

The definition of the FTISS Lyapunov function is given for the global case, the local one can be obtained for $x \in \mathcal{V}$ and $d \in \mathcal{D}$. The first result of this section is as follows.

Theorem 14. If for the system (3) there exists an FTISS (FTiISS) Lyapunov function, then it is FTISS (FTiISS).

Proof. The proof follows from the well known results on ISS/iISS and Hong et al. [2010], Heemels and Weiland [2008], Xiaowu et al. [2010] (skipped for brevity).

\subsection{Homogeneity and ISS for DI}

Finally we are going to show that FTISS and FTiISS follow the homogeneity property (depending on its degree). This result seriously simplifies verification of robustness of FTS, since the homogeneity can be checked applying simple algebraic operations.

For ODE (2) and ISS property some results have been proposed in Ryan [1995], Hong [2001]. An extension of those results for (2) with inclusion of integral ISS (iISS) property has been recently proposed by the authors Bernuau et al. [2013a]. An extension of that result for the DI (3) needs some additional conditions.

Denote an extended discontinuous function

$$
\tilde{F}(x, d)=\left[F_{c l}(x, d)^{T} 0_{p}\right]^{T},
$$

where $0_{p}$ is the zero vector in $\mathbb{R}^{p}$.

Theorem 15. Let the discontinuous function $\tilde{F}$ be homogeneous with the weights $\mathbf{r}=\left[r_{1}, \ldots, r_{n}\right]>0, \tilde{\mathbf{r}}=$ $\left[\tilde{r}_{1}, \ldots, \tilde{r}_{p}\right] \geq 0$ with a degree $\nu \geq-\min _{1 \leq i \leq n} r_{i}$, i.e. $F_{c l}\left(\Lambda_{r} x, \Lambda_{\tilde{r}} d\right)=\lambda^{\nu} \Lambda_{r} F_{c l}(x, d)$. Assume that the system (3) is globally asymptotically stable for $d=0$. Let also

$$
\begin{aligned}
& \left\|F_{c l}(y, d)-F_{c l}(y, 0)\right\|_{H} \leq \sigma(\|d\|) \forall y \in S_{r}, \\
& \sigma(s)= \begin{cases}c s^{\varrho_{\min }} & \text { if } s \leq 1 \\
c s^{\varrho_{\max }} & \text { if } s>1\end{cases}
\end{aligned}
$$

for some $c>0$ and $\varrho_{\max } \geq \varrho_{\min }>0$. Then the system (3) is

ISS if $\tilde{r}_{\min }>0$, where $\tilde{r}_{\min }=\min _{1 \leq j \leq p} \tilde{r}_{j}$;

iISS if $\tilde{r}_{\max } \varrho_{\min }-\mu \leq \nu \leq \tilde{r}_{\min }=0$, where $\tilde{r}_{\max }=$ $\max _{1 \leq j \leq p} \tilde{r}_{j}$.

If $\nu<0$, then under the conditions above the system is FTISS/FTiISS.
Proof. Due to Theorem 8 there exists at least locally Lipschitz continuous and homogeneous Lyapunov function $V: \mathbb{R}^{n} \rightarrow$ $\mathbb{R}_{+}$such that $V\left(\Lambda_{r} y\right)=\lambda^{\mu} V(y)$ for all $y \in S_{r}, \lambda \in \mathbb{R}_{+}$with some $\mu>\max \{1,-\nu\}$ and

$$
\sup _{F_{c l}(y, 0)} D V(y) \psi \leq-a,\|D V(y)\| \leq b \quad \forall y \in S_{r},
$$

$a>0, b>0$. Note that by its definition the function $V$ is positive definite and radially unbounded and $\nu+\mu>0$. We will use the coordinate transformation $x=\Lambda_{|x|} y$, where $\Lambda_{|x|}=$ $\left.\Lambda_{r}\right|_{\lambda=\|x\|_{r}}$, which connects any $x \in \mathbb{R}^{n}$ with the corresponding point $y \in S_{r}$. For the input $d$ we will use the transformation $d=\tilde{\Lambda}_{|x|} \tilde{d}$, where $\tilde{d} \in \mathbb{R}^{p}$ and $\tilde{\Lambda}_{|x|}=\left.\Lambda_{\tilde{r}}\right|_{\lambda=\|x\|_{r}}$, then

$$
\|\tilde{d}\| \leq \rho\left(\|x\|_{r}\right)\|d\|, \rho(s)=\left\{\begin{array}{ll}
s^{-\tilde{r}_{\max }} & \text { if } s \leq 1 \\
s^{-\tilde{r}_{\min }} & \text { if } s>1
\end{array} .\right.
$$

Now let us consider the time derivative of the Lyapunov function $V$ computed for the system (3) for all $x \in \mathbb{R}^{n}$ and $d \in \mathbb{R}^{p}$ :

$$
\begin{gathered}
\sup _{\varphi \in F_{c l}(x, d)} D V(x) \varphi=\|x\|_{r}^{\nu+\mu} \sup _{\phi \in F_{c l}(y, \tilde{d})} D V(y) \phi \\
\leq-a\|x\|_{r}^{\nu+\mu}+\|x\|_{r}^{\nu+\mu} \sup _{\xi \in F_{c l}(y, \tilde{d})-F_{c l}(y, 0)} D V(y) \xi \\
\leq-a\|x\|_{r}^{\nu+\mu}+b\|x\|_{r}^{\nu+\mu} \gamma\left(\|x\|_{r}\right) \sigma(\|d\|),
\end{gathered}
$$

where

$$
\gamma(s)=\sigma \circ \rho(s)=\left\{\begin{array}{ll}
s^{-\tilde{r}_{\max } \varrho_{\min }} & \text { if } s \leq 1 \\
s^{-\tilde{r}_{\min } \varrho_{\max }} & \text { if } s>1
\end{array} .\right.
$$

Therefore if $-\tilde{r}_{\min } \varrho_{\max }<0$, which is equivalent to $\tilde{r}_{\min }>0$, then $\gamma^{-1} \in \mathcal{K}_{\infty}$ and for $\|d\| \leq \sigma^{-1}\left[a /(2 b) \gamma^{-1}\left(\|x\|_{r}\right)\right]$ we have $\sup _{\varphi \in F_{c l}(x, d)} D V(x) \varphi \leq-0.5 a\|x\|_{r}^{\nu+\mu}$ that implies ISS property Heemels and Weiland [2008]. If $\tilde{r}_{\max } \varrho_{\min }-\mu \leq \nu \leq$ $\tilde{r}_{\min } \varrho_{\max }$, then

$$
\|x\|_{r}^{\nu+\mu} \gamma\left(\|x\|_{r}\right) \leq v\left(\|x\|_{r}\right), \quad v(s)= \begin{cases}1 & \text { if } s \leq 1 \\ s^{\mu} & \text { if } s>1\end{cases}
$$

and for the Lyapunov function $W(x)=\ln [1+V(x)]$ (it is locally Lipschitz continuously, positive definite and radially unbounded) we obtain

$$
\begin{aligned}
\sup _{\varphi \in F_{c l}(x, d)} D W(x) \varphi & \leq \frac{\left.-a\|x\|_{r}^{\nu+\mu}+b v\left(\|x\|_{r}\right) \sigma(\|d\|)\right]}{1+V(x)} \\
& \leq-a \frac{\|x\|_{r}^{\nu+\mu}}{1+V(x)}+\tilde{b} \sigma(\|d\|)
\end{aligned}
$$

for some $\tilde{b}>0$, which implies iISS for (3). Since for $\tilde{r}_{\min }>$ 0 the system is ISS (and it is also iISS by the definition), the only interesting admissible value is $\tilde{r}_{\min }=0$. Since $c_{1}\|x\|_{r}^{\mu} \leq V(x) \leq c_{2}\|x\|_{r}^{\mu}$ for $c_{1}=\inf _{y \in S_{r}} V(y)$ and $c_{2}=\sup _{y \in S_{r}} V(y)(V$ is homogeneous with the degree $\mu)$, then for $\nu<0$ we have $\|x\|_{r}^{\nu+\mu} \geq\left[c_{2}^{-1} V(x)\right]^{1+\frac{\nu}{\mu}}$ and the FTS property follows.

Interestingly to note, that FTS and iISS have a similar restriction on the degree of homogeneity: it has to be negative (non positive for iISS).

Corollary 16. If for some homogeneous $F$ (with the degree $\nu$ and the weights $\mathbf{r}$ ) we have $F_{c l}(x, d)=F(x)+d$, i.e. $d$ is an additive disturbance, then the system (2) is ISS if $\nu>-\min _{1 \leq i \leq n} r_{i}$, and it is iISS for $\nu=-\min _{1 \leq i \leq n} r_{i}$.

\section{APPLICATION TO SLIDING MODES}

Let $s: \mathbb{R}^{n} \rightarrow \mathbb{R}$ be a $C^{r}$ function, the time derivatives $s^{(r)}(x):=\left.\frac{d^{k} s(x)}{d t^{k}}\right|_{(1)}, k=1,2, \ldots, r-1$ are continuous func- 
tions of the system state $x \in \mathbb{R}^{n}$ and $s^{(r)}(x):=\left.\frac{d^{r} s}{d t^{r}}\right|_{(1)}$ is the discontinuous one. The set $\mathcal{S}_{r}=\left\{x \in \mathbb{R}^{n}: s(x)=\ldots=s^{(r-1)}(x)=0\right\}$ is said to be the $r$-th order sliding manifold of the system (2) iff it is invariant and locally finite time attractive. The motion of the system (1) on the manifold $S_{r}$ is called by the $r$-th order sliding-mode (r-SM).

\subsection{First order sliding mode control}

Consider the nonlinear system (2) with $d \equiv 0$. Let the sliding manifold be defined by a $C^{1}$ vector-valued function $s: \mathbb{R}^{n} \rightarrow$ $\mathbb{R}^{m}: \mathcal{S}=\left\{x \in \mathbb{R}^{n}: s(x)=0\right\}$.

Let us consider the following switching control:

$$
u=\left(u_{1}, \ldots, u_{m}\right)^{T}: u_{i}=\left\{\begin{array}{l}
u_{i}^{+}(x) \text { if } s_{i}(x)>0 \\
u_{i}^{-}(x) \text { if } s_{i}(x)<0
\end{array},\right.
$$

where $s(x)=\left(s_{1}(x), \ldots, s_{m}(x)\right)^{T}$ and $u_{i}^{+}: \mathbb{R}^{n} \rightarrow \mathbb{R}, u_{i}^{-}$: $\mathbb{R}^{n} \rightarrow \mathbb{R}$ are some functions.

If $s(x)=0$ is the sliding manifold of the system (2), then the invariance condition for the sliding manifold, which provides the equivalent control Utkin [1992], is: $\dot{s}=0 \Leftrightarrow u=u_{e q}(x)$ and the attractivity condition is:

$$
s^{T} \dot{s}<0 \Leftrightarrow \min \left(u_{i}^{+}, u_{i}^{-}\right)<\left(u_{e q}\right)_{i}<\max \left(u_{i}^{+}, u_{i}^{-}\right) .
$$

This condition does not imply that the sliding manifold is reached in finite time. Thus, for the existence of a sliding mode it should be replaced by a more restrictive condition, for example, $s^{T} \dot{s} \leq-\mu\|s\|, \quad \mu>0$. Indeed, one can proves that the Lyapunov function $V(s(t))=\frac{1}{2} s(t)^{T} s(t)$ goes to zero in a finite time (see, Theorem 3 ).

Consider the perturbed control system of the form

$$
\dot{x}=f(x)+G(x)(u+p),
$$

where $x \in \mathbb{R}^{n}, u \in \mathbb{R}^{m}$ and $p \in \mathbb{R}^{m}$ describes bounded matched uncertainties and disturbances. Assume that $\left|p_{i}\right|<\gamma_{i}$, $i=1,2, \ldots, m$ where $\gamma_{i}$ are known positive numbers.

Denote $\mathcal{L}_{f} s(x)=\frac{\partial s}{\partial x} f(x), \mathcal{L}_{G} s(x)=\frac{\partial s}{\partial x} G(x)$. Assume that $\mathcal{L}_{G} s$ is invertible and define the control $u=u(t, x)$ in the form Utkin [1992]

$$
u=-\left(\mathcal{L}_{G} s\right)^{-1} \mathcal{L}_{f} s-(\mu+\|\bar{\gamma}\|)\left(\mathcal{L}_{G} s\right)^{-1} \operatorname{sign}(s),
$$

where $\bar{\gamma}=\left(\gamma_{1}, \ldots, \gamma_{m}\right)^{T}$. The differential equation for the sliding variable has the form

$$
\dot{s}=-(\mu+\|\bar{\gamma}\|) \operatorname{sign}(s)+p .
$$

The Lyapunov function for this system has the form $V(s)=$ $\frac{1}{2} s^{T} s$. Since $\dot{V}=s^{T} \dot{s} \leq-\mu\|s\|$ then the system (10) is uniform FTS (see, Definition 9) with respect to bounded disturbances with a known estimate of a maximum magnitude.

The same Lyapunov function provides the finite time stability of the extended differential inclusion (see, Section 3). Note that

$$
\begin{aligned}
& \dot{s} \in-(\mu+\|\bar{\gamma}\|) \overline{\operatorname{sign}}(s)+\left(\left[-\gamma_{1}, \gamma_{1}\right], \ldots,\left[-\gamma_{m}, \gamma_{m}\right]\right)^{T}, \\
& \overline{\operatorname{sign}}(s)=\left(\overline{\operatorname{sign}}\left(s_{1}\right), \ldots, \overline{\operatorname{sign}}\left(s_{m}\right)\right)^{T}, \\
& \overline{\operatorname{sign}}\left(s_{i}\right)=\left\{\begin{array}{cl}
\{1\} & \text { for } s_{i}>0 \\
\{-1\} & \text { for } s_{i}<0 \\
{[-1,1]} & \text { for } s_{i}=0
\end{array}\right.
\end{aligned}
$$

is homogenous of negative degree. Therefore the system (11) with additional matched disturbances $d \in \mathbb{R}^{m}$

$$
\dot{s} \in-(\mu+\|\bar{\gamma}\|) \overline{\operatorname{sign}}(s)+\left(\left[-\gamma_{1}, \gamma_{1}\right], \ldots,\left[-\gamma_{m}, \gamma_{m}\right]\right)^{T}+d
$$

satisfies Theorem 15 with $\mathbf{r}=[1, \ldots, 1]^{T} \in \mathbb{R}^{m}, \tilde{\mathbf{r}}=[0, \ldots, 0]$, $\nu=-1, \mu=2, \varrho^{\min }=\varrho^{\max }=1$, that provides FTiISS property with respect to matched disturbances of the 1-SM control system.

Remark that the 1-SM system is FTISS with respect to measurement noises. Indeed, for the system

$$
\dot{s} \in-(\mu+\|\bar{\gamma}\|) \overline{\operatorname{sign}}(s+d)+\left(\left[-\gamma_{1}, \gamma_{1}\right], \ldots,\left[-\gamma_{m}, \gamma_{m}\right]\right)^{T}
$$

the Lyapunov function $V\left(s_{i}\right)=\frac{1}{2} s_{i}^{2}, i=1,2, \ldots, m$ gives $\dot{V}_{i}<0$ for $\left|s_{i}\right|>\left|d_{i}\right|$ implying FTISS (see, Theorem 14).

\subsection{Second order sliding mode control}

Let sliding manifold be defined by $\mathcal{S}_{2}=\left\{x \in \mathbb{R}^{n}: s(x)=\dot{s}(x)=0\right\}$.

Assume that the relative degree of $s$ with respect to control input is equal to 2 . In this case the differential equation for the output dynamics has the form

$$
\ddot{s}=a(x)+b(x) u,
$$

where $a: \mathbb{R}^{n} \rightarrow \mathbb{R}$ and $b: \mathbb{R}^{n} \rightarrow \mathbb{R}$. The output and its derivative are assumed to be measured and used for control purposes, i.e. $u=u(s, \dot{s})$.

Under the following assumptions

$$
|a(x)| \leq C, \quad 0<b_{\min } \leq b(x) \leq b_{\max } \quad \forall x \in \mathbb{R}^{n}
$$

the output control system (13) can be extended to the inclusion

$$
\ddot{s} \in[-C, C]+\left[b_{\min }, b_{\max }\right] \bar{u}
$$

where $\bar{u}$ is the set-valued extension of the discontinuous control $u$ obtained in accordance with Filippov definition of the solution for systems with discontinuous right-hand sides (see Section 3).

The "standard" second order sliding mode controllers for the system (13) have the forms Levant [1993]:

$$
u(s, \dot{s})=-k_{1} \operatorname{sign}(s)-k_{2} \operatorname{sign}(\dot{s}),
$$

where $0<k_{2}+C / b^{\min }<k_{1}<\left(\left(b^{\min }+b^{\max }\right) k_{2}-\right.$ $2 C) /\left(b^{\max }-b^{\min }\right)$;

$$
u(s, \dot{s})=-\alpha \operatorname{sign}(z), \quad z=\dot{s}+\beta\lceil s\rfloor^{1 / 2},
$$

where $\alpha, \beta>0, \beta^{2} \leq 2(\alpha-C)$.

Denote $y_{1}=s, y_{2}=\dot{s}$ and present the extended differential inclusion for the system (13) in the form

$$
\left\{\begin{array}{l}
\dot{y}_{1}=y_{2} \\
\dot{y}_{2} \in[-C, C]+\left[b_{\min }, b_{\max }\right] \bar{u}\left(y_{1}, y_{2}\right),
\end{array}\right.
$$

where $\bar{u}$ is one of the controllers (16), (17) with the sign function replaced by its set-valued extension $\overline{\operatorname{sign}}$ (see (12) or Section 3 for the details).

It is easy to see that the system (18) with the controllers (16)(17) is r-homogenous of degree -1 , where $\mathbf{r}=(2,1)^{T}$.

Finite time stability analysis of these 2-SM systems using homogenous Lyapunov function method is presented in Polyakov and Poznyak [2012].

In order to study ISS properties of 2-sm systems let us consider

$$
\left\{\begin{array}{l}
\dot{y}_{1}=y_{2}+d_{1} \\
\dot{y}_{2} \in[-C, C]+\left[b_{\min }, b_{\max }\right] \bar{u}\left(y_{1}, y_{2}\right)+d_{2}
\end{array}\right.
$$

where $d=\left(d_{1}, d_{2}\right)^{T} \in \mathbb{R}^{2}$ are disturbances. It is easy to see that this system with controllers (16) and (17) satisfies conditions of Theorem 15 with $\mathbf{r}=[2,1], \tilde{\mathbf{r}}=[1,0], \nu=$ $-1, c=1, \varrho^{\min }=\varrho^{\max }=1$. That implies FTiISS property for the corresponding closed-loop systems. Moreover, using the 
same theorem we obtain FTISS property of these systems with respect to the first input only, i.e. for $d_{2} \equiv 0$.

Consider the output control system of the form

$$
\dot{s}=a(t)+u,
$$

where the unknown function $a: \mathbb{R} \rightarrow \mathbb{R}$ is assumed to be Lipshitz continuous with the known Lipschitz constant $L>0$.

Let $u$ has the form of the so-called super-twisting controller (STC) Levant [1993] defined for $\alpha>0$ and $\beta>0$ by

$$
u(s(t))=-\alpha\lceil s(t)\rfloor^{1 / 2}-\beta \int_{0}^{t} \operatorname{sign}(s(\tau)) d \tau,
$$

Denoting $x=s$ and $y=a(t)-\beta \int_{0}^{t} \operatorname{sign}(s(\tau)) d \tau$ we can extend the system (20)-(21) to the following DI

$$
\left\{\begin{array}{l}
\dot{x}=-\alpha\lceil x(t)\rfloor^{1 / 2}+y \\
\dot{y} \in-\beta \operatorname{sign}(x)+[-L, L],
\end{array}\right.
$$

where $\overline{\operatorname{sign}}$ is defined by (12) and $\alpha>0$ and $\beta>3 L+2 \frac{L^{2}}{\alpha^{2}}$.

The system (22) is r-homogenous with degree -1 , where $\mathbf{r}=(2,1)^{T}$. The non-smooth homogenous Lyapunov function for finite-time stability analysis of this system is presented in in Moreno [2012]. Using Theorem 15, FTISS and FTiISS properties of the super-twisting system can be shown similarly to (18) with (16) and (17).

ISS analysis of the higher order sliding mode control systems can be done in similar way.

\section{CONCLUSION}

A brief introduction to the theories of homogeneity and finitetime stability is given. The notions of finite-time ISS and iISS are introduced for differential inclusions. Sufficient Lyapunov conditions are formulated. The main result establishes a relation between the degree of homogeneity of a discontinuous system and its ISS or iISS property. The proposed theory is applied to a list of sliding-mode control algorithms.

\section{REFERENCES}

V. Andrieu, L. Praly, and A. Astolfi. Homogeneous Approximation, Recursive Observer Design, and Output Feedback. SIAM J. Control Optimization, 47(4):1814-1850, 2008.

E. Bernuau, A. Polyakov, D. Efimov, and W. Perruquetti. ISS and iISS properties of homogeneous systems. In Proc. of ECC 2013, 2013a.

E. Bernuau, A. Polyakov, D. Efimov, and W. Perruquetti. On extension of homogeneity notion for differential inclusions. In Proc. of ECC 2013, 2013 b.

S. P. Bhat and D.S. Bernstein. Continuous finite-time stabilization of the translational and rotational double integrator. IEEE Trans. Automat. Control, 43(5):678-682, may 1998.

S.P. Bhat and D.S. Bernstein. Geometric homogeneity with applications to finite-time stability. Mathematics of Control, Signals and Systems, 17:101-127, 2005.

S.N. Dashkovskiy, D.V. Efimov, and E.D. Sontag. Input to state stability and allied system properties. Automation and Remote Control, 72(8):1579-1614, 2011.

C. Edwards and S. Spurgeon. Sliding mode control: theory and applications. 1998.

D. Efimov and W. Perruquetti. Oscillations conditions in homogenous systems. In Proc. IFAC NOLCOS Symp., pages 1379-1384, 2010.
A. F. Filipov. Differential Equations with Discontinuous Righthand Sides. Kluwer Academic Publishers, 1988.

L. Grüne. Homogeneous state feedback stabilization of homogeneous systems. SIAM J. Control Optimization, 38(4): 1288-1314, 2000.

V.T. Haimo. Finite time controllers. SIAM J. Control Optim., 24(4):760-770, 1986.

W.P.M.H. Heemels and S. Weiland. Input-to-state stability and interconnections of dicontinuous dynamical systems. Automatica, 44:3079-3086, 2008.

H. Hermes. Nilpotent approximations of control systems and distributions. SIAM journal on control and optimization, 24 : $731,1986$.

H. Hermes. Nilpotent and high-order approximations of vector field systems. SIAM review, 33(2):238-264, 1991.

Y. Hong. $\mathrm{H}_{\infty}$ control, stabilization, and input-output stability of nonlinear systems with homogeneous properties. Automatica, 37(7):819-829, 2001.

Y. Hong, Z.-P. Jiang, and G. Feng. Finite-time input-to-state stability and applications to finite-time control design. SIAM J. Control Optim., 48(7):4395-4418, 2010.

M. Kawski. Nilpotent Lie algebras of vector fields. J. reine angew. Math, page 1, 1988.

A. Levant. Sliding order and sliding accuracy in sliding mode control. International Journal of Control, 58(6):1247-1263, 1993.

A. Levant. Quasi-continuous high-order sliding-mode controllers. IEEE Transactions on Automatic Control, 50(11): 1812-1816, 2005.

J. Moreno. Strict lyapunov functions for the super-twisting algorithm. IEEE Transactions on Automatic Control, 57(4): 1035-1040, 2012.

E. Moulay and W. Perruquetti. Finite time stability of non linear systems. In IEEE Conference on Decision and Control, pages 3641-3646, Hawaii, USA, 2003.

E. Moulay and W. Perruquetti. Finite time stability of differential inclusions. IMA J. Math. Control Inform, 2005.

N Nakamura, H Nakamura, Y Yamashita, and H Nishitani. Homogeneous Stabilization for Input Affine Homogeneous Systems. IEEE Trans. Aut. Contr., 54(9):2271-2275, 2009.

Y. Orlov. Finite time stability and robust control synthesis of uncertain switched systems. SIAM J. Control Optim., 43(4): 1253-1271, 2005.

W. Perruquetti and J. P. Barbot. Sliding Mode Control in Engineering. Marcel Dekker Hardcover, 2002.

A. Polyakov and A. Poznyak. Unified lyapunov function for a finite-time stability analysis of relay second-order sliding mode control systems. IMA Journal of Mathematical Control and Information, page doi: 10.1093/imamci/dns007, 2012.

L. Rosier. Homogeneous Lyapunov function for homogeneous continuous vector field. Systems \& Control Letters, 19:467473, 1992.

E.P. Ryan. Universal stabilization of a class of nonlinear systems with homogeneous vector fields. Systems \& Control Letters, 26:177-184, 1995.

V. I. Utkin. Sliding Modes in Control Optimization. SpringerVerlag, Berlin, 1992.

M. Xiaowu, G. Yang, and Z. Wei. Integral input-to-state stability for one class of discontinuous dynamical systems. In Control Conference (CCC), 2010 29th Chinese, pages 912914, july 2010. 